1 Pak, W. L., Grossfield, J., and White, N. V., Nature, 222, 351 (1969).

2 Hotta, Y., and Benzer, S., Nature, 222, 354 (1969).

3 Cosens, D. J., and Manning, A., Nature, 224, 285 (1969).

4 Heisenberg, M., J. Exp. Biol., 55, 85 (1971).

'Pak, W. L., in Handbook of Genetics (edit. by King, R. C.) (Van Nostrand-Reinhold, New York, in the press).

${ }^{6}$ Alawi, A. A., thesis, Purdue Univ. (1972).

"Alawi, A. A., Jennings, V., Grossfield, J., and Pak, W. L., in The Visual System (edit. by Arden, G. B.), 1 (Plenum, New York, 1972).

${ }^{8}$ Goldsmith, T. H., J. Gen. Physiol., 49, 265 (1965).

${ }^{9}$ Bullock, T. H., and Horridge, G. A., Structure and Function in the Nervous System of Invertebrates, 1063 (Freeman, San Francisco, 1965).

${ }^{10}$ Suzuki, D. T., Piternick, L. K., Hayashi, S., Tarasoff, M., Baillie, D., and Erasmus, U., Proc. US Nat. Acad. Sci., 57, 907 (1967).

11 Suzuki, D. T., Grigliatti, T., and Williamson, R., Proc. US Nat. Acad. Sci., 68, 890 (1971).

12 Jockusch, H., Biochem. Biophys. Res. Commun., 21, 577 (1966).

$1 s$ Ostroy, S. E., and Pak, W. L., Nature New Biol., 243, 120 (1973).

\section{The Choice of Code Words in Phage MS2 RNA}

BALL $^{1}$ has recently reconsidered the question whether degenerate code words are chosen on the basis of their ability to optimize secondary structure. Early evidence by Adams et al. ${ }^{2}$, who sequenced a hairpin of the phage R17 coat protein gene, suggested that third letters of degenerate codons might be selected on the basis of their possible involvement in base pairing.

Working with fragments of the coat protein gene of MS2 we reached tentatively a similar conclusion ${ }^{3}$. Furthermore, we pointed out that the optimal advantage of degeneracy is achieved when third letters are not opposite each other in bihelical regions ${ }^{4}$. When the complete nucleotide sequence of the coat gene became known and the "flower" model was derived for its secondary structure, we no longer found statistically valid evidence for a higher involvement of third letters in base pairing or for a selection in phasing the antiparallel strands of the double helical stems ${ }^{5}$. The latter conclusion was re-emphasized by North ${ }^{6}$.

Ball $^{1}$, however, has divided the (mainly third) codon letters into two option and four option categories. He believes that there is good evidence that four option positions avoid facing each other as base pair partners. The advantage of this phasing is that the multiple option (third) letters can now each form a base pair. In agreement with our earlier conclusions, however, four option letters are not convincingly more involved in base pairing than the others ${ }^{1}$. Also, if selection pressure acts against four option base pairs, would it not decrease the likelihood of two or three option codon letters to face another two, three or four option letter, as this would still considerably benefit the secondary structure?

There are several reasons for believing that the choice between degenerate code words is not random. The selection may be to optimize secondary structure (aspects of our model are still very tentative), or to achieve a complex tertiary structure, or subject to regulations imposed by the translation process, or other requirements still unknown. Also there is, most probably, a strong selection pressure operative at the level of the three dimensional structure of the RNA, as untranslated regions are even less prone to mutation than translated regions ${ }^{7}$. Our present inability to prove that the code degeneracy plays an important role in achieving a specific and functional three dimensional structure of the RNA cannot be taken as proof for constraints on the amino acid sequence, although the latter hypothesis may very well turn out to be correct.

Rijksuniversiteit Gent, W. FIERS

Laboratorium voor Moleculaire Biologie, 900 Gent

Received April 24, 1973.
${ }^{1}$ Ball, L. A., Nature new Biol., 242, 44 (1973).

2 Adams, J. M., Jeppesen, P. G. N., Sanger, F., and Barrell, B. G., Nature, 223, 1009 (1969).

${ }^{3}$ Fiers, W., Contreras, R., De Wachter, R., Haegeman, G., Merregaert, J., Min Jou, W., and Vandenberghe, A., Biochimie, 53, 495 (1971).

- Min Jou, W., Haegeman, G., and Fiers, W., FEBS Lett., 13, 105 (1971).

s Min Jou, W., Haegeman, G., Ysebaert, M., and Fiers, W., Nature, 237, 82 (1972).

- North, A. C. T., Nature new Biol., 239, 76 (1972).

'De Wachter, R., Vandenberghe, A., Merregaert, J., Contreras, R., and Fiers, W., Proc. Natn. Acad. Sci. U.S.A., 68, 585 (1971).

\section{Pharmacological Implications of the Conformation of the Methadone Base}

Methadone (6-dimethylamino-4,4-diphenyl-3-heptanone) is unusual among the potent narcotic analgesics in that it possesses a high degree of conformational flexibility. It has been proposed that the protonated and unprotonated forms of this molecule occur preferentially in cyclic conformations ${ }^{1,2}$, but a crystallographic study ${ }^{3}$ of $\mathbf{S}(+)$-methadone hydrobromide revealed that the protonated form of the molecule, at least in the solid state, prefers an extended (acyclic) conformation. Here we report the conformation of the methadone base observed in crystalline material and its biological implications.

Crystallization of laevo-rotatory methadone from methanol yielded orthorhombic prisms. The unit cell has dimensions, $a=1,685.4, b=1,138.0$, and $c=962.4 \mathrm{pm}$, space group $P 22_{1} 2_{1}$, and contains four molecules $\left(\mathrm{C}_{21} \mathrm{H}_{27} \mathrm{NO}\right)$. Intensities of 1,870 symmetry independent reflexions were measured on an automated Hilger and Watts Y290 diffractometer using graphitemonochromatized MoK $\alpha$ radiation. These intensity data were processed in the usual manner and absorption corrections were deemed unnecessary. The structure was solved by direct methods making use of the multiple solution technique and cyclic application of the tangent formula ${ }^{4,5}$. From an EFourier synthesis, calculated with the phases obtained from the most consistent solution, the coordinates of all the nonhydrogen atoms were derived. Subsequent refinement (least squares using a block-diagonal approximation to the normal equations and difference electron density syntheses) enabled the positional and thermal parameters to be established accurately and all hydrogen atoms to be located. The crystallographic agreement factors at the present stage of refinement are $R=0.040$ and $R_{\mathrm{w}}=0.034$ (for 1,213 reflexions with measured intensity significantly above background). In the following discussion the signs of the torsion angles obtained for the analgetically more active $6 \mathrm{R}(-)$-enantiomer are changed to those of the less potent $6 \mathrm{~S}(+)$-enantiomer in order to permit a direct comparison with crystallographic results on $6 \mathrm{~S}(+)$-methadone hydrobromide ${ }^{3}$.

Table 1 Comparison of Torsion Angles of $6 \mathrm{~S}(+)$-Methadone

\begin{tabular}{lccc}
\hline \multicolumn{1}{c}{ Atoms } & Base & Conjugate acid & $|\Delta|$ \\
$C(1)-C(2)-C(3)-C(4)$ & $164.9^{\circ}$ & $203.3^{\circ}$ & $38.4^{\circ}$ \\
$C(2)-C(3)-C(4)-C(5)$ & -58.5 & 174.1 & 127.4 \\
$C(3)-C(4)-C(5)-C(6)$ & -66.0 & -76.4 & 10.4 \\
$C(4)-C(5)-C(6)-N$ & 69.8 & 146.3 & 76.5 \\
$C(5)-C(6)-N-C(8)$ & -43.2 & -68.6 & 25.4 \\
$C(5)-C(6)-N-C(9)$ & 83.5 & 163.3 & 79.8 \\
$C(6)-C(5)-C(4)-C(10)$ & 59.8 & 50.7 & 9.1 \\
$C(6)-C(5)-C(4)-C(16)$ & 179.8 & 173.7 & 6.1 \\
$C(4)-C(5)-C(6)-C(7)$ & -163.6 & -98.4 & 65.2 \\
$C(5)-C(4)-C(10)-C(11)$ & 79.0 & 79.4 & 0.4 \\
$C(5)-C(4)-C(16)-C(21)$ & 4.3 & 34.0 & 29.7 \\
\hline
\end{tabular}

